\title{
Condicionantes negativos em redes de cooperação: um estudo de caso numa cooperativa agropecuária
}

\author{
Donizeti Leandro SOUZA ${ }^{1}$ \\ Jésus Alex de Lelis MEDEIROS ${ }^{2}$ \\ Lílian FERRUGINI ${ }^{3}$ \\ Cleber Carvalho CASTRO ${ }^{4}$ \\ Márcio SIQUEIRA ${ }^{5}$
}

${ }^{1}$ Graduado em Administração de empresas (UNIVAS), especialista em Gestão de pessoas (UNIFEI) e em Gestão executiva de negócios (PUC-MINAS), atualmente é mestrando em Administração (UFLA) souza.doni@yahoo.com.br.

${ }^{2}$ Graduado em Administração de empresas (UNIVAS), especialista em Gestão executiva de negócios (PUCMINAS), atualmente é Administrador na empresa JR PERSIANAS COUTINHO ME LTDA medeiros_paraiso@hotmail.com.

${ }^{3}$ Graduada em Administração (UFJF), especialista em MBA em Finanças (UFJF), atualmente é mestranda em Administração (UFLA) - lilianufjf@ yahoo.com.br.

${ }^{4}$ Graduado em Administração (UFLA), Mestrado em Administração (UFRGS) e Doutorado em Agronegócio (UFRGS), atualmente é professor adjunto na UFLA e Coordenador Institucional da UAB/UFLA clebercastrouai@gmail.com.

${ }^{5}$ Graduado em Engenharia da Produção (UNIFEI) e Administração (PUC-MINAS), Mestrado (UNIFEI) e Doutorado (USP) em Engenharia de Produção, atualmente é professor adjunto na PUC-Minas Marcio@ comasi.com.br.

\section{Recebido em: 20/05/2013 - Aprovado em: 30/07/2013 - Disponibilizado em: 15/08/2013}

\section{Resumo}

Este estudo tem como objetivo identificar os possíveis condicionantes negativos que contribuem para o enfraquecimento de relações cooperativas, tendo como objeto de investigação, um estudo de caso realizado numa cooperativa agropecuária de Minas Gerais, em que ações individuais dos produtores têm gerado um enfraquecimento nas relações cooperativas, diminuindo a competitividade da rede. Trata-se de um estudo descritivo de caráter qualitativo, sendo a coleta de dados realizada através de questionários (cooperados) e entrevistas em profundidade (dirigentes). Como principais resultados da pesquisa, foram evidenciados os seguintes condicionantes: (1) Insatisfação na política de preços dos produtos; (2) limitação dos serviços prestados aos cooperados; (3) Falta de mobilização e conscientização dos cooperados para com a importância social, e não apenas econômica, que a cooperativa assume enquanto reguladora de mercado e desenvolvimento regional. Estes condicionantes têm contribuído para o enfraquecimento de relações sociais pautadas nos princípios de cooperação, identificando dualidades de interesses individuais / coletivos, além de aspectos econômicos / sociais, articulados através das perspectivas de cooperados e dirigentes. Por fim, espera-se com este estudo estimular a reflexão sobre a necessidade de pessoas/empresas cooperarem para competir, assumindo as cooperativas, um importante instrumento de geração de vantagens competitivas e desenvolvimento regional.

Palavras-chave: Redes de cooperação. Cooperativismo. Relações comportamentais. Condicionantes negativos.

\section{Negative factors in collaborative networks: A case study of an agricultural cooperative}

\begin{abstract}
This study aims at identifying the possible negative factors which contribute to the weakening of cooperative relations, having an investigative objective in the case study of an agricultural cooperative in Minas Gerais, where actions of individual producers have generated a weakening in cooperative relations, decreasing the competitiveness of the network. This is a qualitative descriptive study, with data collection conducted through questionnaires (cooperative) and
\end{abstract}


in-depth interviews (leaders). The main results of the research have shown the following conditions: (1) dissatisfaction when it comes to product pricing, (2) limitation of services to cooperative members, (3) Lack of awareness and mobilization of the cooperative towards social aspects and not only economical ones, which the cooperative assumes as market and regional development regulator. These conditions have contributed to the weakening of social relationships which guided the principles of cooperation, identifying dualities of individual / collective and also economical / social interests, articulated through the perspectives of members and leaders. Finally, we expect this study to stimulate reflection on the need for people / companies to cooperate in order to compete, so that cooperatives became an important tool for generating competitive advantage and regional development.

Keywords: Cooperation networks. Cooperatives. Behavioral relationships. Negative factors.

\section{INTRODUÇÃO}

Nos últimos anos, têm-se observado um aumento expressivo em estudos envolvendo redes de cooperação, surgindo a partir de abordagens multidisciplinares em teorias advindas da economia industrial, custos de transação, dependência de recursos, redes sociais, alianças estratégicas e estudos organizacionais, como estratégia para a criação de vantagens competitivas

(LACOSTE, 2012; CHAUVET et al., 2011; BALESTRIN; VERSCHOORE; REYES JUNIOR, 2010; MA，2004; BORGATTI; FOSTER, 2003; BRITTO, 2002; NOHRIA, 1992; BENGTSON; KOCK, 1999).

Este crescente interesse, segundo Nohria (1992), deve-se a três fatores principais: (1) Amadurecimento do tema na área acadêmica; (2) Aumento da produção e de novas tecnologias; e (3) Nova competição através de relacionamentos horizontais e conexões entre empresas.

No campo de estudos brasileiros, notase uma presença marcante de trabalhos orientados à competitividade e desempenho organizacional. Tendo como objetivos, explorar as estruturas de redes reunindo atributos que permitam uma adequação ao ambiente competitivo, por meio de ações dinâmicas descentralizadas, visando a flexibilidade e o aumento da competitividade no mercado (BALESTRIN; VERSCHOORE; REYES JUNIOR, 2010; NOHRIA, 1992).

Percebe-se que grande parte destes estudos destaca apenas os casos de sucesso, demonstrando os efeitos positivos da organização em rede através de mecanismos de cooperação, negligenciando os casos em que a complexidade de gestão e a dificuldade de alinhamentos estratégicos, geram conflitos, incertezas e riscos de oportunismo, elevando os custos de transação, afetando o comprometimento e o grau de confiança entre os atores participantes (WEGNER; PADULA, 2012; PEREIRA et al., 2010; RING, 1999; NORDIN, 2006).

Neste sentido, o objetivo deste artigo é identificar os possíveis condicionantes negativos que contribuem para o enfraquecimento de relações cooperativas, tendo como objeto de investigação, um estudo de caso realizado numa cooperativa agropecuária de Minas Gerais, em que ações individuais dos produtores têm gerado um 
enfraquecimento nas relações cooperativas, diminuindo a competitividade da rede.

Como forma de atender o objetivo proposto, num primeiro momento será apresentado um quadro teórico sobre redes de cooperação e estruturas cooperativistas, especificando sua contextualização, conceitos e principais perspectivas de estudos. Em seguida será apresentado um estudo empírico realizado numa cooperativa agropecuária de Minas Gerais, investigando os possíveis condicionantes que tem contribuído para o enfraquecimento de relações sociais de cooperação, em dualidades de interesses, de aspectos econômicos e sociais, articulados através das perspectivas de cooperados e dirigentes.

\section{REFERENCIAL TEÓRICO}

\section{Redes de cooperação}

Nos últimos anos, os efeitos da globalização têm forçado as organizações a adotarem modelos de gestão pautados no paradoxo do "cooperar para competir", transcendendo os formatos tradicionais de competição individual para relações coletivas de cooperação, visando à criação de vantagens competitivas (LACOSTE, 2012; BENGTSON; KOCK, 1999;).

Neste sentido, Lacoste (2012), assevera que a maior parte das estruturas de redes cooperativas se forma através de benefícios relacionais, baseados na confiança e comprometimento, ou transacionais, baseados em preços competitivos e ações oportunistas. Quanto mais simples o produto ou o processo de compra, maior a chance de prevalecer os benefícios transacionais de curto prazo, cujo foco recai sobre o preço. Já em produtos ou processos complexos de compra, maiores são as chances de prevalecer os benefícios relacionais, pois o foco passa a ser a relação mútua entre os atores participantes (indivíduos ou organizações) para a criação de vantagens competitivas sustentáveis no longo prazo. Desta forma, fatores relacionais tende a favorecer uma relação cooperativa, já os fatores transacionais tendem a gerar relações competitivas de preço. Surge assim, o conceito de "coopetição", nas situações em que os atores buscam desenvolver benefícios relacionais e transacionais na busca pela sinergia de esforços entre os fatores de cooperação e competição em relações interorganizacionais híbridas (LACOSTE, 2012).

Uma das preocupações das redes de cooperação tem sido o desafio de encontrar respostas estratégicas a esta dualidade de interesses, administrando riscos, incertezas e ações oportunistas, além de reduzir os custos de transação para tornar as redes mais eficientes. Deste desafio surge a necessidade de esforços organizacionais na identificação, persuasão e sinergia de indivíduos, produzindo relações de confiança e governabilidade em que as decisões de cooperação possam ser modeladas, evitando 
conflitos e facilitando a troca de recursos e coordenação de esforços coletivos (CHAUVET et al., 2011; BORGATTI; FOSTER, 2003; NOHRIA, 1992).

Embora seja praticamente impossível evitar conflitos, uma abordagem interativa e reflexiva sobre a implementação de uma rede de cooperação envolve a participação e conscientização de funcionários, clientes e parceiros na moderação de conflitos e seus efeitos negativos, surgindo a necessidade de antecipar falhas de cooperação associados com: (1) o âmbito das alianças formadas, (2) as consequências das relações com o cliente, e (3) o processo de implementação. Quando estas três categorias de conflitos são gerenciadas adequadamente, é provável que os efeitos positivos dos mesmos sejam otimizados em processos de aprendizagem e tomadas de decisão a favor dos efeitos negativos. Porém quando estes fatores são negligenciados, conflitos podem dificultar a implementação e a gestão de estrutura de cooperação (NORDIN, 2006).

Dentre os principais pontos negativos dos conflitos gerados em redes, EklinderFrick; Eriksson e Hallén (2012) destacam as seguintes consequências: (1) possibilidade de bloqueio da rede a novas informações; (2) perda do foco econômico coletivo; (3) difícil gerencimento de estruturas de poder; (4) criação de subgrupos isolados, capazes de gerar insatisfação de outros agentes participantes; e (5) baixa relevância de informações e participação na rede. Assim, redes de cooperação devem ser analisadas como uma moeda de duas faces, que podem apresentar vantagens e desvantagens em sua estrutura social.

Neste contexto, será apresentada na próxima seção, uma análise nas estruturas cooperativas como um tipo de redes de cooperação, destancando as abordagens econômicas e comportamentais nas estruturas relacionais e transacionais de coordenação.

\section{Estruturas cooperativistas}

O Cooperativismo como movimento socioeconômico é antigo, tendo suas origens na Inglaterra em 1844 com a Sociedade Rochdale dos pioneiros equitativos, em que o êxito de suas ações frente ao capitalismo fizesse com que os ideais cooperativistas proporcionasse grande expansão na Europa e posteriormente para o resto do mundo (GAWLAK; RATZKE, 2010; CANÇADO, 2007; SINGER, 2002; HIND, 1997).

Nos dias atuais, o cooperativismo tem despertado a atenção de políticos e estudiosos pela importância econômica e social que assume no desenvolvimento regional da sociedade, tornando-se uma temática importante para investigar níveis mais sofisticados de competitividade, em especial aos pequenos produtores ou empresas, seja no acesso a recursos (tangíveis e intangíveis) ou a clientes, acessíveis na maioria das vezes, apenas às grandes empresas com alto poder de 
barganha (CAPALDO, 2007; BALESTRIN; VARGAS; FAYARD， 2005; PROVAN; HUMAN, 1999).

Percebe-se, pela literatura, que o cooperativismo assume duas funções principais: (1) Econômica: na geração de riquezas através de movimentos coletivos entre atores sociais; e (2) Social: sendo um importante instrumento regulador de mercados, pois sua atuação visa garantir os interesses dos agentes envolvidos e proporcionar vantagens competitivas através de negociações coletivas (LAGO; SILVA, 2012). Nesta perspectiva, pode-se verificar que as cooperativas se diferem dos demais modelos organizacionais pelos benefícios e impactos sociais gerados na comunidade, pela forma democrática de gestão e por representar um importante instrumento de desenvolvimento regional através da sinergia de esforços de seus membros.

Segundo Lacoste (2012), as formas de cooperação podem ser estudadas através de relações híbridas de interesses econômicos e sociais dos atores (indivíduos/empresas), que combinam cooperação e competição em aspectos mutuamente opostos, explicados por três mecanismos fundamentais de relacionamento: (1) quando a cooperação desempenha um papel central; (2) quando a competição assume uma posição central; e (3) quando a cooperação e a competição desempenham papéis iguais, refletindo a tensão entre a criação de valor (cooperação) e apropriação de valor (competição).

Desta forma, é comum encontrar na literatura duas abordagens de estudos relacionados às cooperativas: (1) a abordagem econômica, no qual os relacionamentos são baseados em interesses financeiros, através de comportamentos oportunistas e fortes proteções formais e legais; e (2) a abordagem comportamental, focada no compromisso, na coordenação, na interdependência e na confiança, sendo estes importantes pilares para a sobrevivência da mesma, além de representar uma perspectiva mais viável a processos de cooperação como instrumento de vantagens competitivas sustentáveis (LAGO; SILVA，2012; JONES，2004; BARNEY; HANSEN, 1994).

Assim, o cooperativismo assume um modelo de gestão dependente da ajuda mútua de seus membros, visando à união de esforços em prol de um objetivo coletivo comum, atingindo resultados dificilmente alcançáveis se atuando isoladamente. Porém, nem sempre a coletividade se sobrepõe à individualidade no movimento cooperativista, fator este que contribui negativamente para a perda de competitividade devido a falhas nas relações comportamentais de compromisso e confiança (OCEMG, 2011; SCOPINHO, 2007).

\section{METODOLOGIA}

Como etapa complementar à fundamentação teórica, foi realizado um 
estudo descritivo junto a uma cooperativa do setor agropecuário de Minas Gerais, aqui denominada de COOPERAGRO para preservar a identidade dos respondentes. O propósito do estudo é entender os aspectos econômicos / comportamentais de cooperados e cooperativa, na tentativa de buscar respostas sobre os motivos que têm levado a falhas nos processos de cooperação.

$\mathrm{O}$ método escolhido para a pesquisa foi o estudo de caso, que segundo Gil (2002, p. 54) "consiste no estudo profundo $e$ exaustivo de um ou poucos objetos, de maneira que permita seu amplo e detalhado conhecimento, tarefa praticamente impossível mediante outros delineamentos existentes". Já a técnica utilizada para o levantamento de dados foi o uso de questionários, justificado pela facilidade de levantamento dos dados, preservação da identidade dos agentes pesquisados e pela comodidade que esta técnica oferece em questões relativas à disponibilidade de tempo e influência da pessoa do pesquisador, o que garante maior participação e dados mais realistas.

A coleta dos dados ocorreu no ano de 2012, obtendo uma taxa de resposta de $34 \%$ do total de cooperados. Sendo a pesquisa complementada com entrevistas realizadas com dirigentes da cooperativa, visando identificar os condicionantes negativos da cooperação através de diferentes percepções. Trata-se, portanto de um estudo descritivo de caráter qualitativo, cujo objetivo é levantar possíveis condicionantes comportamentais que afetam a relação cooperativista.

\section{Resultados e discussão}

Após análise dos dados coletados, identificou-se que a maioria dos cooperados são moradores antigos da região (entre 12 a 67 anos) e atuam há bastante tempo no setor (entre 15 a 30 anos). Do total de cooperados respondentes, $(78,26 \%)$ participam da cooperativa desde sua fundação e entre todos os entrevistados (100\%) parece haver uma relação boa/muito boa com a equipe atual de dirigentes da cooperativa. Entre os motivos que levaram os produtores a participarem da cooperativa, destacam-se: Indicação de amigos ou familiares (30\%); visualização de melhores oportunidades de negócios (48\%); garantia de comercialização (13\%); melhoria nos processos das técnicas de cultivo $(4,5 \%)$ e outros $(4,5 \%)$.

Por estes dados, percebe-se um alto vínculo dos cooperados com a comunidade local, os quais se mostram muito dependentes do setor de produção para a economia da região, além de um alto índice de aprovação dos cooperados com a cooperativa e seus dirigentes, o que reflete no reconhecimento sobre a importância socioeconômica da cooperativa para a região, seja através da comercialização de produtos, geração de empregos, melhores oportunidades de negócios, garantias de comercialização e infraestrutura oferecida ao produtor. 
Entretanto, após entrevistas iniciais com dirigentes da cooperativa, foi identificado certo conflito de interesses individuais dos cooperados através das seguintes ações: (1) venda direta de parte da produção a atravessadores; (2) baixo envolvimento em assuntos gerenciais (assembléias e reuniões); e (3) desvalorização dos serviços ofertados pela cooperativa, como na compra de insumos, defensivos agrícolas e produtos complementares ofertados.

Assim, ao serem questionados se participam de assembléias ou reuniões, (87\%) os cooperados afirmam participar com freqüência. Contudo, quando questionados sobre a avaliação de participação dos demais cooperados, $(48 \%)$ dos entrevistados afirmam haver pouco interesse e participação dos demais cooperados em assuntos e/ou produtos relacionados à cooperativa, apontando para uma divergência de percepções sobre a participação ativa dos mesmos na cooperativa.

Ao serem questionados sobre os motivos da venda de parte da produção a atravessadores (terceiros), (57\%) afirmam ser o preço o fator mais importante, seguido por (17\%) que afirmam ser o horário de retirada dos produtos e (9\%) que afirmam ser outros fatores relacionados à comercialização. Completando a pesquisa (17\%) dos entrevistados não responderam a questão.

Segundo depoimentos de dirigentes da cooperativa, grande parcela dos produtores se preocupa em entregar apenas a cota mínima da produção estabelecida no estatuto, negociando grande parte dela com atravessadores, situação esta agravada principalmente por grandes produtores, no qual foram identificados percentuais baixos de produção destinados à cooperativa (entre $20 \%$ a 50\%), diferente dos pequenos produtores que afirmam destinar de $(80 \%)$ a (100\%) da produção.

Segundo Jones (2004); Barney e Hansen (1994), a perspectiva comportamental pautada na confiança, comprometimento e maior interação informal de cooperados se mostram uma alternativa mais viável para se criar vantagens competitivas em relação à perspectiva econômica, visão esta não identificada nos dados levantados, pois percebe-se uma relação mais econômica, ao invés de comportamental dos cooperados, caracterizando fatores transacionais baseados em preços e ações oportunistas (LACOSTE, 2012; LAGO; SILVA, 2012).

Identificam-se, ainda, relações de cooperação baseadas fortemente em contratos formais (cota mínima), desviando parte dos princípios cooperativistas de valores mutualistas, comprometimento e confiança como instrumentos de criação de vantagens competitivas (OCEMG, 2011; VIZEU, 2009; SEBRAE, 2009; SCOPINHO, 2007).

Quando indagados sobre a avaliação dos principais serviços prestados pela cooperativa, a política de preços e a comercialização dos produtos apresentaram os 
percentuais de aprovação mais baixos em relação a outros benefícios pesquisados como: insumos, defensivos agrícolas, suporte de máquinas, melhorias de plantios, dentre outros. Atingindo um percentual de apenas (30\%) de aprovação como boa, fator este que explica uma das causas da venda de parte da produção a atravessadores.

Complementando a pesquisa, os cooperados foram questionados sobre a avaliação geral dos serviços prestados, assim como os possíveis benefícios que gostariam que fossem ofertados pela cooperativa. A maioria dos entrevistados (48\%) afirmou que gostaria que a cooperativa agregasse maior valor aos cooperados através da melhoria da infraestrutura (acessos a serviços de máquinas e auxílio de plantio), planos de saúde, dentre outros benefícios sugeridos; (43,5\%) gostariam que fossem criadas estratégias que melhorassem os preços de comercialização dos produtos, como melhorias de negociação com compradores ou beneficiamento da produção e; por fim $(8,5 \%)$ afirmaram estarem satisfeitos com os serviços prestados.

No quadro 1 são apresentados os principais condicionantes identificados na pesquisa, assim como as possíveis sugestões de melhoria sob a ótica dos entrevistados:

Quadro 1 - Principais condicionantes negativos evidenciados na pesquisa

\begin{tabular}{|c|l|}
\hline $\begin{array}{c}\text { Melhoria dos serviços } \\
\text { ofertados }\end{array}$ & $\begin{array}{l}\text { Na percepção dos cooperados, a cooperativa poderia agregar maior valor aos serviços } \\
\text { prestados, seja na melhoria de acesso a máquinas e equipamentos, apoio a pequenos } \\
\text { produtores, maior auxílio na aração/plantio e benefícios exclusivos, como: brindes, } \\
\text { descontos em insumos e defensivos agrícolas, planos de saúde e treinamentos. }\end{array}$ \\
\hline $\begin{array}{c}\text { Política de } \\
\text { preços/lucros }\end{array}$ & $\begin{array}{l}\text { A venda direta de parte da produção a atravessadores tem gerado certa insatisfação da } \\
\text { cooperativa quanto à filosofia cooperativista. Sob a percepção dos cooperados, isto ocorre } \\
\text { devido à política de preços praticada pela cooperativa em relação aos atravessadores, dos } \\
\text { quais sugerem melhor negociação de preços, garantias de comercialização, maior } \\
\text { participação nos lucros e formas alternativas de agregação de valor ao produto para preços } \\
\text { mais competitivos. }\end{array}$ \\
\hline $\begin{array}{c}\text { Conscientização dos } \\
\text { cooperados }\end{array}$ & $\begin{array}{l}\text { Por fim, um ponto importante evidenciado na pesquisa, se refere à necessidade de } \\
\text { conscientização e mobilização dos cooperados quanto aos princípios cooperativistas. } \\
\text { tem contribuído para caracterizar relações mais econômicas e formais (cota mínima) ao } \\
\text { invés de relações comportamentais de confiança e benefícios mútuos, gerando o } \\
\text { enfraquecimento de laços mais fortes de cooperação e competitividade. }\end{array}$ \\
\hline
\end{tabular}

Fonte: Elaborada pelos autores

\section{CONSIDERAÇÕES FINAIS}

Fundamentados nos aspectos teóricos e empíricos apresentado, identificaram-se os possíveis condicionantes que contribuem negativamente para as relações cooperativas. Percebe-se haver uma falha de conscientização dos cooperados sobre a importância social, e não apenas econômica, 
que a cooperativa assume na regulação de mercado e desenvolvimento regional.

A falta desta mobilização tem gerado perspectivas de interesses individuais, ao invés de coletivos, o que tem contribuído para conflitos nas relações entre cooperativa e cooperados, sendo os fatores: preços e interesses pessoais, importantes causas de descontentamento dos cooperados.

Nota-se que este descontentamento tem contribuído para um enfraquecimento de relações cooperativistas, evidenciando baixo comprometimento dos cooperados, principalmente na venda de parte da produção a atravessadores, que como possuem custos menores (compra direta) conseguem negociar diretamente com os produtores através de preços mais atrativos.

Esta postura tem gerado um viés de debate, pois fatores individualistas e econômicos parecem contrariar os princípios cooperativistas pautados em benefícios mútuos e sociais, contribuindo para o enfraquecimento nas relações de confiança dos agentes envolvidos.

Frente às limitações da pesquisa, sugere-se a partir deste estudo, novas pesquisas específicas sobre os condicionantes apresentados neste artigo, além de pesquisas sobre o desenvolvimento de possíveis alternativas de gestão capazes de inibir a ações de atravessadores e aumentar os níveis comportamentais entre cooperativa e cooperados, seja através de novos processos de negociação ou agregação de valor dos produtos comercializados.

Por fim, espera-se com o artigo ter atingido o objetivo inicial ao identificar os possíveis condicionantes que têm contribuído aos desvios de valores cooperativistas, tendo como objeto de investigação uma cooperativa agropecuária. Percebeu-se que esta possui falhas nas estratégias de cooperação, contribuindo para a ampliação e reflexão do debate sobre a necessidade de cooperação como uma importante estratégia de competição, não apenas sobre a ótica econômica, mas principalmente social e que as cooperativas podem assumir enquanto instrumento de desenvolvimento regional.

\section{REFERÊNCIAS}

BALESTRIN, A.; VARGAS, L. M.; FAYARD, P. Ampliação interorganizacional do conhecimento: o caso das redes de cooperação. REAd, v. 11, n. 1, p. 1-25, 2005.

BALESTRIN, A.; VERSCHOORE, J. R.; REYES JÚNIOR, E. O campo de estudo sobre rede de cooperação interorganizacional no Brasil. RAC, v. 14, n. 3, p. 458-477, 2010.

BARNEY, J. B.; HANSEN, M. H.

Trustworthiness as a source of competitive advantage. Strategic Management Journal, Sussex, v. 15, p. 175-190, 1994, Special issue.

BENGTSON, M.; KOCK, S. Cooperation and competition in relationships between competitors in business networks. Journal of business \& industrial marketing. v.14, n.3, p.178-193, 1999.

BORGATTI, S. P.; FOSTER, P. The network paradigm in organizational research: a review 
and typology. Journal of Management. n.29, v.6, p. 991-1013, 2003.

BRITTO, J. Cooperação interindustrial e redes de empresas. In: KUPFER, D.; HASENCLEVER, L. (Org.) Economia industrial: fundamentos teóricos e práticas no Brasil. Rio de Janeiro: Campus, 2002.

CANÇADO, A. C. Autogestão em Cooperativas populares: Os desafios da prática. 1 ed. Salvador: IES, 2007.

CAPALDO, A. Network structure and innovation: the leverage of a dual network as a distinctive relational capability. Strategic Management Journal, v. 28, p. 585-608, 2007.

CHAUVET, V.; CHOLLET, B.; SODA, G.; HUAULT, I. The contribution of network research to managerial culture and practice. European Management Journal. v. 29, p. 321-334, 2011.

EKLINDER-FRICK, J.; ERIKSSON, L. T. HALLÉN, L. Effects of social capital on processes in a regional strategic network. Industrial Marketing Management, v.41, p. 800-806, 2012.

GAWLAK, A.; RATZKE, F. A.

Cooperativismo: primeiras lições. 4. ed. Brasília: Sescoop, 2010.

GIL, A. C. Como elaborar projetos de pesquisa. 4 ed. São Paulo: Atlas, 2002.

HIND, A. M. The Changing Values of the Cooperative and Its Business Focus.

American Journal of Agricultural

Economics, v. 79, n. 4, p. 1077-1082, 1997,

JONES, E. C. Wealth-Based Trust and the Development of Collective Action. World Development v.. 32, n. 4, p. 691-711, 2004.

LACOSTE, S. "Vertical coopetition": the key account perspective. Industrial Marketing Management, v.41, p. 649-658, 2012.
LAGO, A.; SILVA, T. N. Condicionantes do desenvolvimento de relacionamentos intercooperativos no cooperativismo agropecuário. Organizações Rurais \& Agroindustriais, v. 14, n. 2, p. 212-226, 2012.

MA, H. Toward global competitive advantage: creation, competition, cooperation, and co-option. Management decision.v.42, n.7, p. 907-924, 2004.

NOHRIA, N. Is a network perspective a useful way of studying organizations? In: NOHRIA, N.; ECCLES, R. G. Networks and organizations: structure, form, and action. Boston: Harvard Business School Press, 1992.

NORDIN, F. Identifying intraorganisational and interorganisational alliance conflicts: a longitudinal study of an alliance pilot project in the high technology industry. Industrial marketing management. v. 35, 2006.

OCEMG/SESCOOP. Informações Econômicas e Sociais do cooperativismo mineiro. Sistema OCEMG/SESCOOP-MG, Belo Horizonte: Bigráfica editora, 2011.

PEREIRA, B. A. D.; VENTURINI, J. C.; WEGNER, D.; BRAGA, A. L. Desistência da cooperação e encerramento de redes interorganizacionais: em que momento essas abordagens se encontram. Revista de Administração e Inovação. v.7, n.1, 2010.

PROVAN, K. G.; HUMAN, S. E. Organizational learning and the role of the network broker in small-firm manufacturing.In: GRANDORI, A. Interfirm networks: organization and industrial competitiveness. London: Routledge, 1999.

RING, P. S.; The costs of networked organization. In: GRANDORI, A. Interfirm networks: organization and industrial competitiveness. London: Routledge, 1999.

SCOPINHO, R. A. Sobre cooperação e cooperativas em assentamentos rurais. Psicol. Soc., v. 19, n. spe, 2007. 
SERVIÇO BRASILEIRO DE APOIO ÀS

MICRO E PEQUENAS EMPRESAS.

Cooperativa de crédito. Série

empreendimentos coletivos, Brasília:

SEBRAE, 2009.

SINGER, P. Introdução à economia

solidária. São Paulo: Fundação Perseu

Abramo, 2002.

VIZEU, F. Contribuições da sociologia da dádiva aos estudos sobre organizações substantivas. O\&S, v.16 - n.50, p. 409-427, 2009.

WEGNER, D.; PADULA, A. D. Quando a cooperação falha: um estudo de caso sobre o fracasso de uma rede interorganizacional.

Revista de Administração da Mackenzie. v. 13, n.1, p.145-171, 2012. 